\title{
Effects of the Duration of the Electric Test Pulse upon the Zeta-Value of the Human Retina
}

\author{
By
}

\author{
Takeshi Onoki, Eiichi Onodera \\ (小野木 健) (小野寺栄一) \\ and Hiroshi Yamamoto \\ (山本 弘志) \\ From the Physiological Laboratory of Prof. K. Motokawa, \\ Tohoku University, Sendai
}

(Received for publication, February 23, 1956)

\section{INTRODUCTION}

Motokawa invented $\left.{ }^{1-2}\right)$ a new method for analyzing retinal processes concerning chromatic and achromatic vision. The method consists in measuring variations in electrical excitability of the eye after exposure to light. The aspects of variations depend on the wave-length of preillumination. When red, yellow, green and blue lights are used for preillumination, the eye becomes most sensitive to the electrical test stimulus 1, 1.5, 2 and 3 seconds respectively after termination of the pre-illuminating light. The rod ${ }^{3-4)}$ or scotopic process has a maximum of its electrical excitability at 4.5 seconds from the onset of the light stimulus. Tukahara and $A \mathrm{Ab}^{5}$ ) investigated the relation between retinal processes and the stimulating frequency of the repetitive rectangular pulses after a white pre-illumination applied to the dark-adapted eye, and found that the frequencies $20,36,42$, and $55 \mathrm{cps}$. were resonance frequencies for the rod process, blue-, green- and red processes respectively.

Motokawa and $\left.\mathrm{Ebe}^{6}\right)$ studied strength-frequency relation in stimulation experiments of the eye with sinusoidal a.c., and found several minima in the strength-frequency curve. They further showed that the electrical sensitivity to a. c. of $30-37,40-45,47-55$ and $60-100$ cps. was enhanced selectively by blue, green, yellow and red lights respectively. Yonemura and Nango ${ }^{71}$ measured the strength-duration curve of the darkadapted eye and compared it with that of the light-adapted eye. When the illumination was low the ratio of the electric threholds of the lightand the dark-adapted eyes was found maximal at a duration of about 20 msec. From this finding they concluded that the optimal stimulating duration for the rod process would be about $20 \mathrm{msec}$.

Takahashi, Fujimaki and Yoshino ${ }^{8}$ stimulated the eye by sinusoidal 
a. c. in a partially illuminated state of the retina. They obtained several minima in the strength-frequency curve similar to those found by Motokawa and Ebe.

In the present experiment we studied the effects of the duration of rectangular pulses which were used as test stimuli in.determination of retinal processes.

\section{EXPERTMENTAL}

\section{Method}

The method consists in measuring the electrical excitability of the eye after a brief exposure to light.

After a preliminary dark adaptation of about 20 minutes, the eye was exposed to a white test patch of $1^{\circ}$ in visual angle, then the eye was stimulated with a rectangular single pulse of varying duration. The stimulating current of varying duration ranging from 3 to $200 \mathrm{msec}$. was obtained by means of a spring rheotome.

The electrical threshold was measured in such a way, that starting with a voltage sufficient to cause a distinct light sensation, the stimulating voltage was lowered in gross steps at first and in smaller steps towards the threshold. Near the threshold comparison procedures were used which consisted in comparison of the effects of the test stimulus and the control stimulus of zero voltage. The index used was the least perceptible light sensation. The difference between the values of electrical excitability (reciprocals of thresholds) with and without pre-illumination was expressed in percentage of the latter, and denoted by $\zeta . \quad \zeta=100\left(\mathrm{E}-\mathrm{E}_{\mathrm{o}}\right) / \mathrm{E}_{\mathrm{o}}$, where $E$ and $E_{o}$ represent electrical excitability determined with and without pre-illumination. In this way, the $\zeta$ values were measured $1,1.5,2$, or 3 seconds after the end of pre-illumination. $\zeta$ values were plotted against the time after termination of pre-illumination. Curves so obtained were called excitability curves.

\section{Results}

1) Excitability curves determined by stimulating currents of varying durations

Motokawa ${ }^{1-2)}$ showed that the shape of the excitability curves obtained by pre-illumination with colored lights were characteristic of the wave-lengths. That is, when red, yellow, green and blue lights were used for pre-illumination, the maxima of the excitability curves lie at $1,1.5,2$ and 3 seconds respectively. In the present experiment, the central region of the retina was illuminated with white light of 100 lux for a period of 2 seconds, and then the electrical excitability was tested 
at $1,1.5,2$ or 3 seconds with a single pulse of duration ranging from 3 to $200 \mathrm{msec}$.

In Fig. 1, $\zeta$-time curves or excitability curves determined with pulses of different durations are illustrated. The number of each curve represents the duration of the stimulating current, and the zero-level for each curve is indicated on the left side.

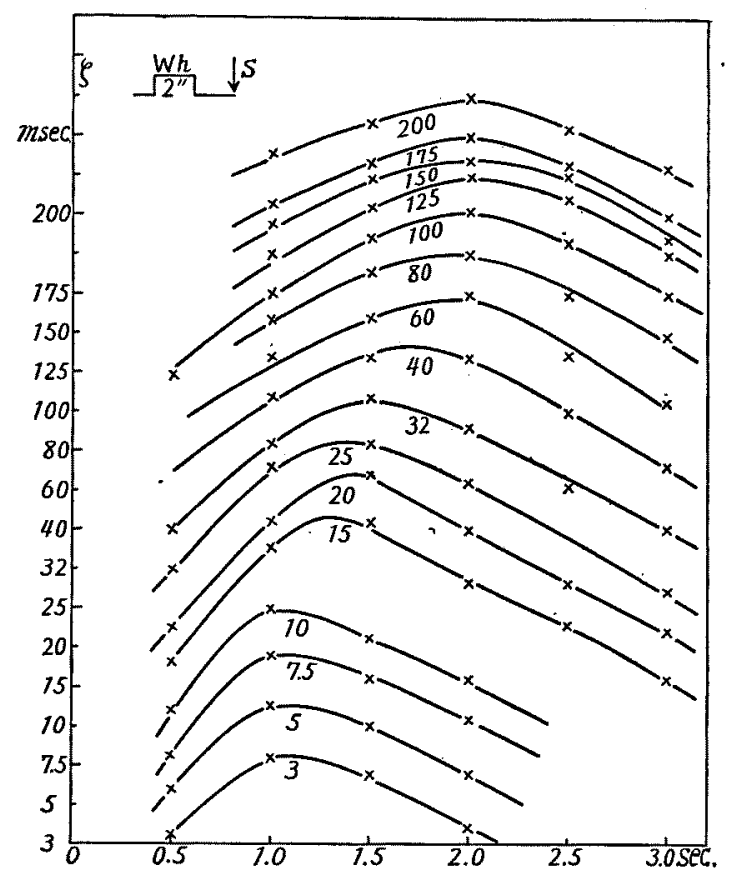

Fig. 1. Excitability curves determined with single test electric pulses of varying durations after the fovea was exposed to light (100 lux). Abszissae : time in seconds after termination of light, Ordinates : $\zeta$ values. Number of each curve represents duration of stimulating current, and zero-level for each curve is indicated on left side.

As can be seen in Fig. 1, the curves for durations of 200 to $60 \mathrm{msec}$. have a crest time of about 2 seconds. As the stimulating duration was decreased, the crest time was shortened by and by from 2 seconds to 1.5 second. The curves for durations of 32-15 msec. have their maxima at 1.5 second. These curves are the same in shape as a curve which would be obtained after yellow pre-illumination. When the stimulating duration is shorter than $10 \mathrm{msec}$. the curves show their maxima at 1 second, and are in shape similar to a curve which would be obtained by red pre-illumination. It seems that pulses of shorter duration are more favorable for eliciting the red process. 
In Fig. 2, $\zeta$-values at 1, 1.5, 2 and 3 seconds of each excitability curves are plotted as ordinates against durations of stimulating cur-

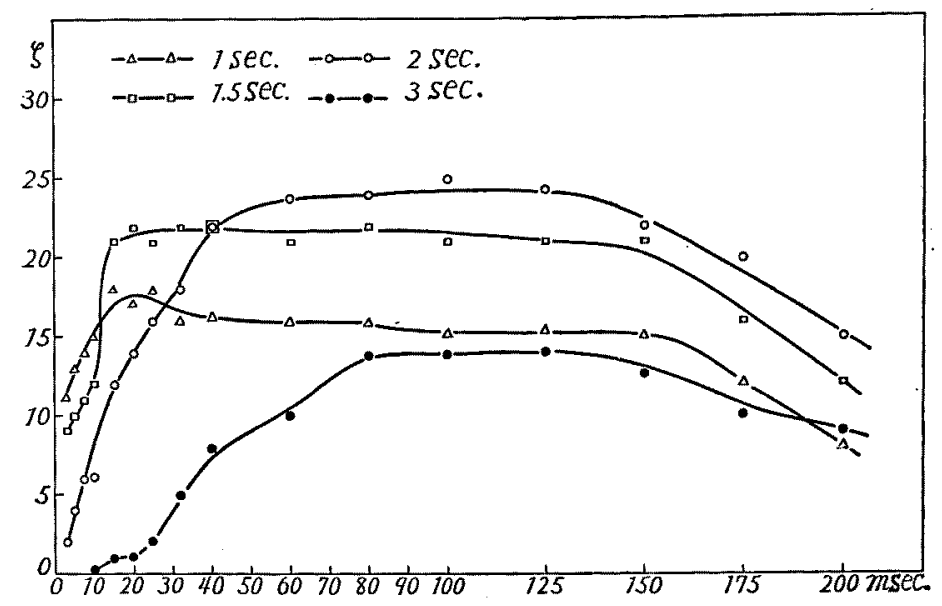

Fig. 2. $\zeta$-duration curves for photopic processes.

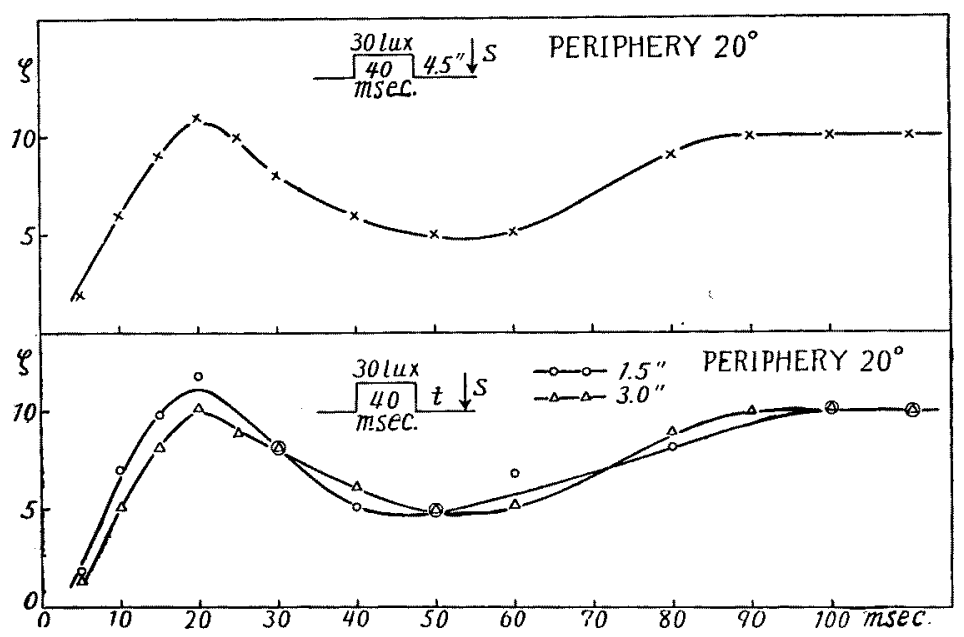

Fig. 3. $\zeta$-duration curves for scotopic processes obtained at $20^{\circ}$ from fovea after a white flash of 30 lux and $40 \mathrm{msec}$. Upper curve : $\zeta$-duration curve for rod process. Lower set of curves: $\zeta$-duration currves for twin process.

rents as abscissae. The curves run parallel when the stimulating durations are long enough. As the duration was shortened, the curve for 3 seconds began to decrease at about $80 \mathrm{msec}$., and the curve for 2 seconds at $60 \mathrm{msec}$. The curve for 1.5 second is higher than the others over 32 
to $15 \mathrm{msec}$, but the curve for 1 second is predominant for durations shorter than $10 \mathrm{msec}$. These results are alike to the relation of $\zeta$ values to the stimulating frequency of repetitive stimulation which was studied by Tukahara \& Abe. The time constants of color receptor systems must be greater in the order, blue $>$ green $>$ yellow $>$ red, and this assumption conforms not only to our results obtained by single stimulation, but also to Tukahara-Abe's results obtained by repetitive stimulation.

2) Excitablity of the scotopic processes

Motokawa and his co-workers ${ }^{3-4}$ ) showed that the rod process has a crest time of its excitability at 4.5 seconds from the onset of a light stimulus. Oikawa $^{9}$ ' found that scotopic vision is mediated not only by the rod process but also by his so-called twin process. This process consists of a complementary pair of yellow and blue processes and subserves white sensation at low illuminations like the rod process. The crest times of the two components of the twin process are 1.5 and 3 seconds. We used 30 lux white light instead of 100 lux in this experiment. A retinal area at $20^{\circ}$ from the fovea was exposed to a white light for a period of $40 \mathrm{msec}$. and then $\zeta$ values at $1.5,3$ and 4.5 seconds were measured.

$\zeta$-duration curves are illustrated in Fig. 3. The upper curve in this figure refers to the rod process and the lower two curves refer to the twin process.

As can be seen in this figure, these 3 curves are very similar to one another not only in shape but also in values of $\zeta$. As the stimulating duration became shorter, $\zeta$ values decreased gradually, reached a minimum at 50 to $40 \mathrm{msec}$., and then increased to make a maximum at about 20 msec. From this fact, it may be said that the stimulating duration of about $20 \mathrm{msec}$. is most effective for exciting scotopic processes, while those of 50 to $40 \mathrm{msec}$. are least effective.

In Fig. 4 , the $\zeta$ values are represented as a function of the intensities of conditioning flashes. Electric pulses of 20 and $100 \mathrm{msec}$. were used because of their effectiveness for the scotopic processes. As is shown in this figure, the curves for 20 and $100 \mathrm{msec}$. run parallel. As the light intensity rises by and by from complete darkness, the $\zeta$ value increases gradually to reach its maximal value at about 3 lux, remains almost constant till about $50 \mathrm{lux}$, and then decreases steeply. This finding indicates that the optimal intensity for the scotopic processes is about 3 lux. The depression of $\zeta$ values at higher intensities was interpreted by Oikawa as due to inhibition by the cone processes.

\section{Discussion}

The previous authors ${ }^{5-6}$ ) showed that the frequency of stimulating currents is important for the study of retinal processes, because it enables 


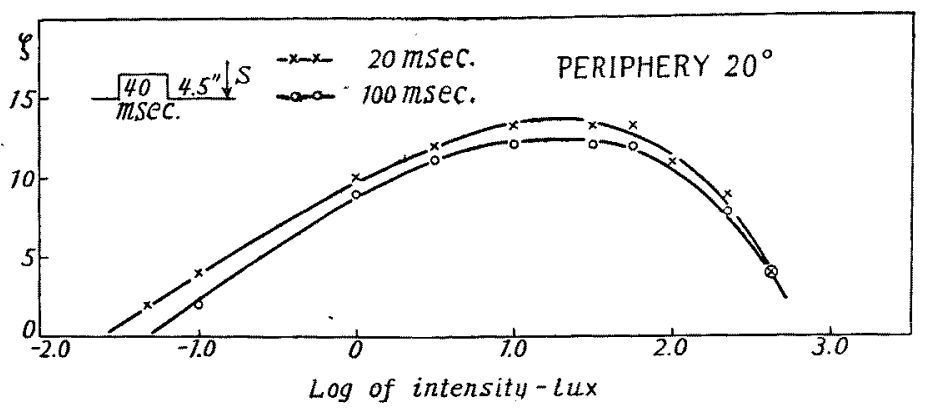

Fig. 4. $\zeta-\log$ intensity curves for rod process obtained at $20^{\circ}$ from fovea with test shocks of 20 and $100 \mathrm{msec}$. in duration after exposure of 40 msec. flash of white light.

selective stimulation of color receptors. As mentioned above, the red receptor has the smallest time constant and the blue one the greatest. The series, red, yellow, green and blue manifests itself in various phenomena such as crest times, reasonance frequencies, etc. Our findings have added important evidence for the theory of time constants of color receptors. When a pulse of about $10 \mathrm{msec}$. in duration is used, an excitability curve (Fig. 1) similar to the red curve is obtained. This is because the red receptor is more strongly excited by a pulse of such short duration. With a pulse of $25 \mathrm{msec}$. a curve similar to the yellow curve is obtained, because the yellow receptor is most sensitive to the pulse of this duration. In this way, selective stimulation of various color receptors would be possible merely by varying the duration of the test pulse. The $\zeta$-duration curves in Fig. 2 not only clarify the ground for the selective stimulation, but also provide the theoretical grounds for Motokawa's method of electrostimulation of the eye, in which single test pulses of $100 \mathrm{msec}$. are usually used. All curves in Fig. 2 run parallel to one another over a range from 80 to $125 \mathrm{msec}$. This means that the $\zeta$ values are independent of the duration of the test pulse over this range, and that electric stimuli have no such selective action upon the color receptors within this range as in the region of shorter durations. We owe the color-effects revealed by Motokawa's method to the use of an electric test pulse of about $100 \mathrm{msec}$,, which is uniformly effective upon all sorts of color receptors. If a pulse of shorter duration, say, of $10 \mathrm{msec}$. were used we would always obtain an excitablity curve having a maximum at $1 \mathrm{sec}$., whatever the color of the pre-illuminating light may be. A pulse of about $20 \mathrm{msec}$. is most favorable for exciting the rod process. This result is in line with that by Yonemura \& Nango. ${ }^{71}$ It is, however, to be noted that a pulse of about $100 \mathrm{msec}$. is nearly as effective as the pulse of $20 \mathrm{msec}$. Therefore it may be said that in Motokawa's routine method test electric pulses act non- 
selectively upon the rod system as well.

\section{SuMmary}

The electrical excitability of the dark-adapted eye was measured by a single pulse of varying durations after exposure to light.

The effect of the light was expressed by $\zeta$, percentage increase of electrical excitability over the resting level.

1. After the eye was illuminated by white light for two seconds, $\zeta$ values were maximal $1,1.5$, and 2 seconds respectively after termination of the light stimulus as the duration of test pulse was 3-10, 20-32, and 60$200 \mathrm{msec}$. In comparison with the data obtained with colored pre-illuminations and a sufficiently long electric test pulse the result was so interpreted that the red, the yellow, and the green receptors were selectively excited by the pulses of $3-10 \mathrm{msec}$, by those of $20-32 \mathrm{msec}$. and by those of $60-200$ msec. respectively.

2. $\zeta$ values measured at $1,1.5,2$ and 3 seconds after termination of the white light were plotted against the duration of the test pulse, and it was found that $\zeta$ values were independent of the duration of the test pulse over a range from $80-125$ msec.

It is likely that the selectively excitatory action of an electric pulse upon color receptors is much weaker in the range of duration around $100 \mathrm{msec}$. than in the range of shorter durations.

3. The dependence of the scotopic processes upon the duration of the test pulse was studied, and it was found that the duration of about $20 \mathrm{msec}$. was most favorable for the rod process in agreement with the data by Yonemura and Nango.

Prof. K. Motokawa furnished guidance and helpful criticism, for which we express here hearty thanks.

\section{References}

1) Motokawa, K., J. Neurophysiol., 1949, 12, 291.

2) Motokawa, K., Tohoku J. Exp. Med., 1949, 51, 165.

3) Motokawa, K. \& Ebe, M., ibid., 1951, 54, 215.

4) Motokawa, K., Ebe, M., Arakawa, Y. \& Oikawa, T., J. Opt. Soc. Amer., 1951, 41, 478.

5) Tukahara, S., \& Abe, Z., Tohoku J. Exp. Med., 1951, 54, 189.

6) Motokawa, K., \& Ebe, M., Science, 1952, 116, 92.

7) Yonemura, O. \& Nango, R., Nihongankagakkai Zasshi (Jap.), 1953, 57, 195.

8) Takahashi, R., Fujimaki, N. \& Yoshino, T., Iwate Ishi (Jap.), 1955, 7, 181.

9) Oikawa, T., Tohoku J. Exp. Med., 1953, 58, 69. 